Original Article

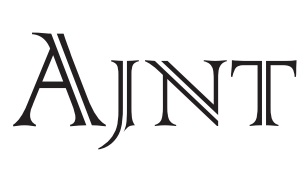

\title{
Acute Urinary Tract Infections in Children in Khartoum State: Pathogens, Antimicrobial Susceptibility and Associated Risk Factors
}

\author{
Eltigani MA Ali ${ }^{\mathrm{a}^{*}}$ and Amira Hassan Osman ${ }^{\mathrm{b}}$ \\ a. Pediatric Department, Soba University Hospital, Khartoum, Sudan \\ b. Pediatric Department Gafar Ibn Auf Children Hospital, Khartoum, Sudan
}

\begin{abstract}
Introduction: Adequate treatment of acute urinary tract infections (UTIs) in children depends on knowledge of the local pattern of causative pathogens and associated risk factors. We explored these patterns and risk factors in a group of Sudanese children who attended the emergency department of Gaafar Ibn Auf Pediatric Hospital in Khartoum, Sudan, with symptoms of acute UTI.
\end{abstract}

Methods: Urine culture and sensitivity testing was performed for symptomatic children who had positive urine dipstick tests for nitrates and leuckocyte esterase.

Results: Acute UTI was confirmed in 100 children, $74 \%$ of them were below 5 -years of age and $35 \%$ were infants. The male-female ratio of affected children was 2.1:1 among infants, and 1:1.2 among older children. Uncircumcision and infancy were significantly more prevalent among children confirmed to have UTI compared to children with non-signifiacnt bacteruria. E. coli was the most commonly isolated pathogen $(60 \%)$. Mean susceptibility of all isolates was high to gentamicin (96\%), ciprofloxacin (94\%), ceftriaxone $(90 \%)$, and cefixime $(85 \%)$. Mean susceptibility was moderate to cefuroxime (75\%), nalidixic acid $(74 \%)$, and nitrofurantoin $(70 \%)$, and low to cephelexin $(51 \%)$, cotrimoxazole (26\%), amoxicillin-clavulanate (19\%) and ampicillin (14\%). Ultrasound scan was feasible in 89 children with confirmed UTI, revealing renal stones in six children $(6.7 \%)$. Micturating cysto-urethrogram (MCUG) was indicated for 28 children but was feasible for only 15 children, revealing low-grade vesico-ureteric reflux (VUR) in five of them.

Conclusion: E. coli was the commonest causative organism of acute UTI in our setting. Isolated pathogens were highly resistant to conventional empiric therapy. Male uncircumcision was significantly associated with UTI among the study patients.

\footnotetext{
* Corresponding Author; Pediatric department, Soba University Hospital, University of Khartoum, PO Box 102, Khartoum, Sudan
}

Keywords: Acute Urinary Tract Infections, Children, Pathogens, Antimicrobial susceptibility, Sudan

\section{Introduction}

Urinary tract infection (UTI) is a common disease in pediatric practice. Early diagnosis and prompt treatment can reduce the risk of renal scarring and its long-term sequelae such as hypertension and end stage renal failure $[1,2]$.

Adequate treatment of acute UTI depends on knowledge of the local pattern of causative pathogens, their antimicrobial resistance and the associated underlying risk factors. The changing pattern of antimicrobial susceptibility of bacterial pathogens causing acute UTI is a growing problem. Sudan is a third world country in which drug prescription is not under strict control. Consequently, many organisms including those causing acute UTI may ultimately develop high resistance to many antibiotics in current use. Moreover, organisms not known to be common in causing acute UTI may emerge as important causative pathogens. Therefore, the knowledge of the local pattern of urinary pathogens and their susceptibility to various antimicrobials are essential for selection of the appropriate empiric therapy for children with acute UTI.

This study was conducted with the aim of studying potential risk factors and local patterns of causative organisms and antimicrobial susceptibility in Sudanese children with acute UTI in order to evaluate new options for empiric therapy.

\section{Methods}

This is a prospective hospital based study conducted in the emergency department of Gaafar Ibn Auf Pediatric Hospital in Khartoum, Sudan. This emergency department serves a large population of children in Khartoum State and its suburbs, and accepts children in the age range of one day to sixteen years. Admitted 
Table 1: Clinical characteristics of children with positive leukocyte esterase test, whose urine samples were submitted to culture

\begin{tabular}{lllll}
\hline Characteristic & & UTI group & No UTI group & P value \\
\hline Age & $<1$ year & $35(35 \%)$ & $22(18.2 \%)$ & 0.008 \\
& $1-4$ years & $39(39 \%)$ & $50(40.5 \%)$ & $49(41.3 \%)$ \\
5-16 years & $26(26 \%)$ & $67(54.5 \%$ & 0.8 \\
Gender & Male & $53(53 \%)$ & $54(45.5 \%)$ & 0.04 \\
Male circumcision & Female & $47(47 \%)$ & $40(60.6 \%)$ & 0.35 \\
No & Yes & $42(77.8 \%)$ & $26(39.4 \%)$ & $12(10 \%)$ \\
History of UTI & Yes & $12(22.2 \%)$ & $109(90 \%)$ & 0.057 \\
Constipation & No & $14(14 \%)$ & $6(5 \%)$ & $116(95 \%)$ \\
\\
Pinworm infestation & Yes & $86(86 \%)$ & $6(5 \%)$ & $115(95 \%)$ \\
\end{tabular}

children are then transferred to a number of tertiary care hospitals in Khartoum for further management, including Soba University Hospital. The researcher attended the emergency department between July and November 2007, recruiting children who presented with symptoms suggestive of acute UTI, until 400 children were included. Children on recent antibiotics therapy and children known to have urinary tract anomalies and/or stones were excluded.

All cases were initially screened by urine dipstick testing (nitrate and leuckocyte esterase) and urine microscopy. Urine samples were collected using mid stream method in toilet-trained children and using clean-catch methods or sterile bladder catheter in younger children and infants. Urine samples from dipstick positive cases were then sent for culture and in vitro sensitivity testing. Culture was done on Cystine Lysine Electrolyte Deficiency (CLED) media with semi-quantitative testing. Culture was considered positive if a single organism was isolated in the presence of a bacterial growth of more than 100,000 $\mathrm{CFU} / \mathrm{ml}$. Sensitivity was tested using the gel diffusion technique on Muller and Hinton agar.

Ultrasound scan was requested for all culture positive patients. Based on the American Academy of Pediatric guidelines, micturition cystourithrogram (MCUG) was requested for children confirmed to have UTI if they were in the age range of 2 months to 2 years, or had family history of vesico-ureteric reflux (VUR), or had ureteric dilatation, pelvi-calyceal dilatation (PCD) or hydronephrosis on ultrasound scan (USS) [1].

Ethical approval was obtained from the University of Khartoum ethical committee. The researchers obtained verbal consent from the parents of each child before inclusion in the study.

Data was analysed using the Statistical Package of Social Science (SPSS) computer program version 15.0. Proportions were compared using Chi square test; $\mathrm{P}$ values $<0.05$ were considered statistically significant.

\section{Results}

Out of 400 children suspected of having acute UTI, 221 $(55.2 \%)$ had positive urine dipstick testing for nitrate and leuckocyte esterase, i.e. bacteruria, and their urine samples were sent for culture. Of these, 100 patients $(45.2 \%)$ had significant growth on urine culture and fulfilled the criteria for the diagnosis of acute UTI. Children confirmed to have UTI had a mean age of 3.4 years (SD 3.6, range 15 days - 14 years), and infants constituted $35 \%$ of them. The majority of children with UTI were males (53\%), the majority of whom were below 5 years of age $(74 \%)$. The male-female ratio of affected children was 2.1:1 among infants, and 1:1.2 among older children. Uncircumcision was more prevalent among children confirmed to have UTI $(77.8 \%$ versus $60.6 \%, \mathrm{P}=0.04)$ than children with non-signifiacnt bacteruria. Young age ( $<1$ year) was also more prevalent in children comfirmed to have UTI than 
Figure 1: Frequency of different pathogens isolated from urine of children with acute UTI $(\mathrm{N}=100)$

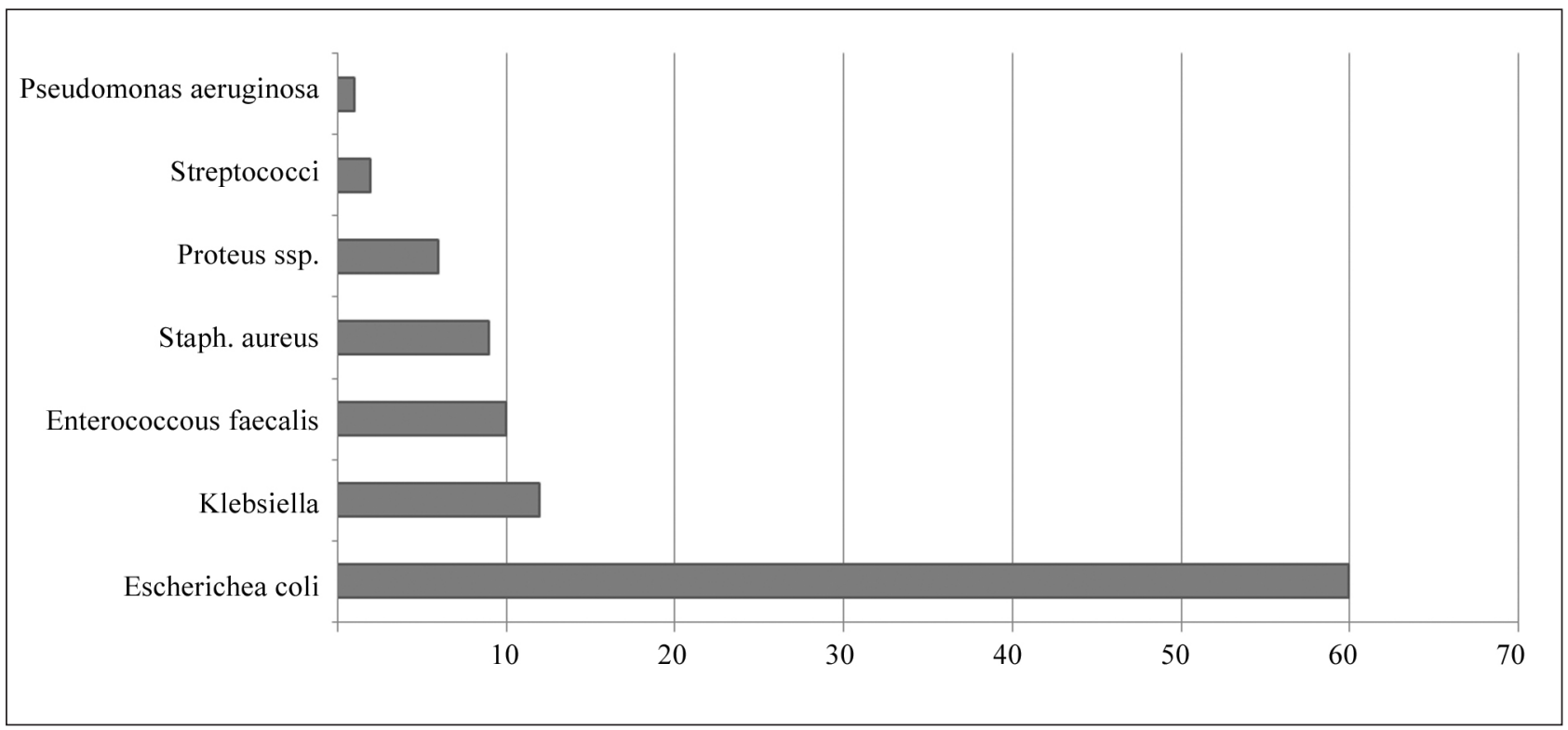

children with non-significant bacteruria (35\% versus $18.2 \%, P=0.008)$. Gender, history of UTI, constipation and pinworm infestation were not significantly different between the two groups (Table 1).

Among culture positive specimens, E. coli was the most commonly isolated pathogen $(60 \%)$, followed by other Gram-negative organisms like Klebsiella (12\%) and Enterococcus faecalis (10\%) (Figure 1).

In vitro sensitivity testing showed that the mean susceptibility of the isolated pathogens was high to gentamicin (96\%), ciprofloxacin (94\%), ceftriaxone $(90 \%)$, and cefixime $(85 \%)$, while it was moderate to cefuroxime $(75 \%)$, nalidixic acid $(74 \%)$, and nitrofurantoin $(70 \%)$, and low to cephelexin (51\%), cotrimoxazole $(26 \%)$, amoxicillin-clavulanate $(19 \%)$ and ampicillin (14\%). E. coli had similar pattern of antibiotics susceptibility (Tables 2-3).

USS was feasible in 89 out of 100 children with confirmed UTI (89\%). Twenty-nine children had abnormal USS findings (32.6\%); six children had renal stones and 23 children had variable urinary tract dilatations.

Because of some difficulties it was not possible to do micturating cysto-urethrogram (MCUG) for all patients in whom these tests were indicated. MCUG was indicated for 28 children, but was feasible in only 15 children. MCUG was done 4-6 weeks after the diagnosis of UTI. The results showed low-grade (I-II) vesico-ureteric reflux (VUR) in five children; three females and two males. DMSA scan was not done for any child.

\section{Discussion}

The predominance of females among children affected with acute UTIs in this study, except during infancy, is consistent with many previous reports [3-6].

The role of male circumcision in preventing UTI in male infants is controversial. Some studies have suggested that circumcision protects against or prevents recurrence of symptomatic UTI in male infants [7]. However, the American Academy of Pediatrics Task Force on Circumcision considered such data insufficient to recommend routine neonatal circumcision [8]. In this series, uncircumcision was significantly more prevalent among children confirmed to have UTI compared to children with non-signifiacnt bacteruria. This finding suggests that uncircumcision may predispose male children to acute UTIs, and is supported by previous studies among Sudanese children [9-11].

Shaikh et al conducted a meta-analysis to determine the pooled prevalence of urinary tract infection (UTI) in children by age, gender, race, and circumcision status. They showed that among febrile male infants less than 3 months of age, $2.4 \%$ of circumcised males and $20.1 \%$ of uncircumcised males had a UTI [12]. In another metaanalysis of 12 studies on male circumcision, including one randomized controlled trial, it has been shown that circumcision was associated with a significantly reduced risk of UTI with an odds ratio of 0.13 [13]. However, given the low risk of UTI in normal boys, the authors concluded that clinical benefit is likely only in boys at high risk of UTI [13]. 
Table 2: Mean antibiotic susceptibility of isolated pathogens in children with acute UTI $(n=100)$

\begin{tabular}{lll}
\hline Antibiotic & Frequency & Percentage (\%) \\
\hline Gentamicin & 96 & 96 \\
Ciprofloxacin & 94 & 94 \\
Ceftriaxone & 90 & 90 \\
Cefixime & 85 & 85 \\
Cefuroxime & 75 & 75 \\
Nalidixic acid & 74 & 74 \\
Nitrofurantoin & 70 & 70 \\
Cephelexin & 51 & 51 \\
Co-trimoxazole & 26 & 26 \\
Amoxcillin-clavulanate & 19 & 19 \\
Ampicillin & 14 & 14 \\
\hline
\end{tabular}

In this series of Sudanese children, E. coli was the commonest pathogen causing acute UTI, accounting for $60 \%$ of cases. This conforms to previous reports from Sudanese children $[9,10,11]$ as well as reports from other parts of the world [3, 6, 14-16].

Isolates causing UTI in children were resistant to cotrimoxazole, ampicillin and amoxicillin-clavulonate in our series, which is consistent with previous reports from Sudan [9-11] as well as reports from other countries $[3,6,14-16]$. The high resistance to amoxicillinclavulanate shown in this and other studies reflecs the growing resistance of urinary pathogens to conventional antibiotics, but alarmingly, resistance seems to be emerging even to recently introduced antibiotics.

Susceptibility was high to gentamicin, ciprofloxacin, and third generation cefalosporins, conforming to data from Tunisia, Turkey, Iran and Taiwan [3, 4, 14, 15]. Therefore, we suggest the use of gentamicin and ceftriaxone as first-line parenteral empiric therapy for acutely ill children with UTI. Cefixime can be used for those who can tolerate oral medication [17]. Moderate susceptibility to nalidixic acid and nitrofurantoin is consistent with reports from Yemen [6], although high sensitivity was reported from Turkey [14].

Among the 15 children who underwent MCUG, results showed low grade VUR in five children $(5 \%)$. This may not reflect the true prevalence of VUR in our setting since an MCUG was done for only half the children in whom it was indicated. However, this prevalence is much lower than figures reported in the literature, whereby $30-35 \%$ of children presenting with their first acute UTI were found to have VUR [18].

\section{Conclusion}

E. coli was the commonest causative organism of acute UTI in our setting. Isolated pathogens were highly resistant to conventional empiric therapy such as first generation cephalosporine, cotrimoxazole, ampicillin and amoxicillin-clavulanate. With the exception of male uncircumcision and infancy, no other risk factors were significantly associated with UTI among patients subjected to urine culture. It is difficult to conclude on the contribution of VUR to acute UTI in our series because of the small number of patients investigated.

\section{Aknowledgements}

This work is part of a Thesis submitted for partial fulfilment of Clinical MD degree in Pediatrics, University of Khartoum (2007). We thank the staff in Gaafar Ibn Auf Pediatric Emergency Hospital and The National Health Laboratory for their help and cooperation.

\section{References}

1. [No authors listed]. Practice parameter: the diagnosis, treatment, and evaluation of the initial urinary tract infection in febrile infants and young children. American Academy of Pediatrics. Committee on Quality Improvement. Subcommittee on Urinary Tract Infection. Pediatrics. 1999;103(4 Pt 1):843-52. Erratum in: 2000;105(1 Pt 1):141. Pediatrics 1999;103(5 Pt 1):1052, 1999;104(1 Pt 1):118.

2. Hellersteim S. Urinary tract infection. Old and new concepts. Pediatr Clin North Am. 1995;42(6):1433-57.

3. Ghedira Besbes L, Messaoudi A, Ben Meriem C, Guediche MN. Profile of antimicrobial resistance of 
Table 3: Antibiotic susceptibility of $\boldsymbol{E}$. coli in children with acute UTI $(n=60)$

\begin{tabular}{lll}
\hline Antibiotic & Frequency & Percentage (\%) \\
\hline Gentamicin & 60 & 100 \\
Ceftriaxone & 59 & 98.3 \\
Cefixime & 59 & 98.3 \\
Ciprofloxacin & 57 & 95 \\
Nalidixic acid & 48 & 80 \\
Nitrofurantoin & 47 & 78.3 \\
Cefuroxime & 44 & 73.3 \\
Cephelexin & 29 & 48.3 \\
Co-trimoxazole & 13 & 21.7 \\
Ampicillin & 3 & 5 \\
Amoxcillin-clavulanate & 2 & 3.3 \\
\hline
\end{tabular}

agents causing urinary tract infections in children. Tunis Med. 2004;82(3):299-305.

4. Sharifian M, Karimi A, Tababaei SR, Anvaripour N. Microbial sesitivity pattern in urinary tract infections in children: a single center experience of 1,177 urine cultures. Jpn Infectious Dis. 2006;59(6):380-2.

5. Qureshi AM. Organisms causing urinary tract infections in pediatric patients at Ayub Teaching Hospital Abbottabad. J Ayub Med Coll Abbottabad. 2005;17(1):72-4.

6. Mohanna MA, Raja $>$ a YA. Frequency and treatment of UTI in children subjected to urine culture, in Sana $>$ a Yemen. J Ayub Med Coll Abbottabad. 2005;17(2): 20-2.

7. Nayir A. Circumcision for the prevention of significant bacteruria in boys. Pediatr Nephrol. 2001 Dec;16(12):1129-34. Erratum in: Pediatr Nephrol 2002;17(4):307.

8. [No authors listed]. Circumcision Policy Statement. American Academy of Pediatrics. Task Force on Circumcision. Pediatrics. 1999;103(3):686-93.

9. Mohamed AB. Study of urinary tract infection in symptomatic under five Sudanese children [MD Thesis]. [Khartoum]: University of Khartoum; 1994. p. 34-40.

10. Omer BE. Urinary tract infection in Sudanese children with severe malnutrition [MD Thesis]. [Khartoum]: University of Khartoum; 2000. p. 55-59.

11. Gadalla YM. Urinary tract infection in febrile infants in Emergency Department in Khartoum State [MD Thesis]. [Khartoum]: University of Khartoum; 2002. p. 70-74.
12. Shaikh N, Morone NE, Bost JE, Farrell MH. Prevalence of urinary tract infection in childhood: a meta-analysis. Pediatr Infect Dis J. 2008;27(4):302-8.

13. Sing-Grewal D, Macdessi J, Craig J. Circumcision for the prevention of urinary tract infection in boys: a systematic review of randomised trials and observational studies. Arch Dis Child. 2005;90(8):853-8.

14.Yüksel S, Oztürk B, Kavaz A, Ozçakar ZB, Acar B, Güriz H, Aysev D, Ekim M, Yalçinkaya F. Antibiotic resistance of urinary tract pathogens and evaluation of empirical treatment in Turkish children with urinary tract infection: Int J Antimicrob Agents. 2006;28(5):413-6.

15. Wu CY, Chiu PC, Hsieh KS, Chiu CL, Shih CH, Chiou YH. Childhood urinary tract infection: a clinical analysis of 597 case. Acta Pediatr Taiwan. 2004;45(6):328-33.

16. Hernández-Porras M, Salmerón-Arteaga G, MedinaSantillán R. Microbial resistance to antibiotics used to treat infections in Mexican children: Proc West Pharmacol Soc. 2004;47:120-2.

17. Hoberman A, Wald ER, Hickey RW, Baskin M, Charron M, Majd M, Kearney DH, Reynolds EA, Ruley J, Janosky JE. Oral versus initial intravenous therapy for urinary tract infections in young febrile children. Pediatrics. 1999 Jul;104(1 Pt 1):79-86.

18. Jacobson SH, Hansson S, Jacosson B. Vesico-ureteric reflux: occurrence and long term risks. Act Pediatr Suppl. 1999;88(431):22-30. 